\title{
Philosophical Aspects of Quantum Field Theory: II
}

\author{
Laura Ruetsche*
}

University of Michigan

\section{Abstract}

According to a regnant criterion of physical equivalence for quantum theories, a quantum field theory (QFT) typically admits continuously many physically inequivalent realizations. This, the second of a two-part introduction to topics in the philosophy of QFT, continues the investigation of this alarming circumstance. It begins with a brief catalog of quantum field theoretic examples of this non-uniqueness, then presents the basics of the algebraic approach to quantum theories, which discloses a structure common even to 'physically inequivalent' realizations of a QFT. Finally, it introduces and evaluates a handful of strategies for interpreting quantum theories in the face of the non-uniqueness of their Hilbert space representations.

Part I of this mathematically informal introduction to interpretive questions that arise in connection with quantum field theory (QFT) highlighted a signal disanalogy between ordinary non-relativistic quantum mechanics (QM) and what it called $\mathrm{QM}_{\infty}$. Ordinary QM lies within the scope of the Stone-von Neumann and Wigner-Jordan theorems, which guarantee the uniqueness of the representation of the canonical commutation relations (CCRs) (Stone von-Neumann) or canonical anticommutation relations (CARs) (Wigner-Jordan) constituting the quantum theory of a system with finitely many degrees of freedom. For $n$ finite, the Jordan-Wigner theorem assures us, any representation of the CARs for $n$ spin systems is unitarily equivalent to any other. Mutatis mutandis (and with a few qualifications (cf. Summers 2001), the Stone-von Neumann theorem assures us that representations of the CCRs are unique up to unitary equivalence. As unitary equivalence is both a plausible and a venerable criterion for the physical equivalence of quantum theories, these theorems are taken to establish that the physical theory obtained by carrying out the instruction 'quantize this system' is unique, provided the system possesses only finitely many degrees of freedom.

Theories of $\mathrm{QM}_{\infty}$ concern systems possessing infinitely many degrees of freedom: QFTs, which associate a field amplitude and a conjugate momentum with every point of spacetime, and the thermodynamic limit of statistical mechanics, reached by letting the number of microsystems considered and the volume they occupy go to infinity, while keeping their density finite. Theories of $\mathrm{QM}_{\infty}$ fall outside the scope of the Stone-von Neumann and Jordan-Wigner theorems. They admit presumptively physically inequivalent realizations. This essay considers interpretive responses to that circumstance. Section 1 documents examples of unitarily inequivalent quantizations of the simplest imaginable classical field theory, the Klein-Gordon theory, and considers repercussions of this non-uniqueness for the idea that QFT - understood by its practitioners as particle physics - sustains a particle interpretation. The consideration is continued in Section 2's account of Haag's theorem and interacting QFTs. Section 3 sketches the rudiments of the algebraic approach to quantum theories, an approach which enables us not only to 
compare different unitarily inequivalent realizations of a quantum theory, but also to see what those realizations might have in common. Section 4 uses the algebraic apparatus to articulate, and begin to evaluate, a variety of approaches to interpreting $\mathrm{QM}_{\infty}$.

\section{The Free Klein-Gordon Field: Particle Ontology}

The theory of the mass $m$ free boson field, obtained by quantizing the classical mass $m$ Klein-Gordon field in Minkowski spacetime, may be the simplest QFT there is. The aim of section's impressionistic treatment is to place this theory of $\mathrm{QM}_{\infty}$ outside the scope of the Stone-von Neumann theorem.

First, some spacetime preliminaries (for a proper introduction, consult Wald 1984) Minkowski spacetime, the spacetime of Special Relativity, is highly symmetric. It admits a (indeed, many) coordinate system such that the light cone structure at each point assumes the familiar $45^{\circ}$ shape in that coordinate system. Less pictorially put, the metric of Minkowski spacetime is unchanged under a group of transformations that include translations, rotations, and Lorentz boosts (which can be thought of as translating between inertial coordinate systems adapted to observers moving at constant velocity with respect to one another). These symmetries of the metric, aka isometries, form a group called the Poincaré group. General Relativity traffics in more general spacetimes, typically with a much more variable light cone structure. An arbitrary general relativistic spacetime - a solution to Einstein's field equations - needn't have any isometries whatsoever.

Where $\left(x_{1}, x_{2}, x_{3}, t\right)$ are inertial coordinates for Minkowski spacetime, the classical Klein-Gordon equation for mass $m$ is

$$
\left(\frac{\partial^{2}}{\partial x_{1}^{2}}+\frac{\partial^{2}}{\partial x_{2}^{2}}+\frac{\partial^{2}}{\partial x_{3}^{2}}-\frac{\partial^{2}}{\partial t^{2}}-m^{2}\right) \varphi(x)=0 .
$$

Solutions $\phi(x)$ to (1) are maps from points of Minkowski spacetime to $\mathbb{C}$. To follow the Hamiltonian recipe for quantizing classical Klein-Gordon theory is to find a Hilbert space representation of CCRs involving the field amplitudes $\varphi(x)$ and conjugate momenta $\pi(x)$ (see Wald (1994, \$3.1) for details).

In Minkowski space-time, such a representation can be obtained by following a textbook 'frequency-splitting' heuristic for quantizing Klein-Gordon theory (for which, see Kaku 1993, Ch. 3.2; Wald 1994, Ch. 4.3). Solutions to the Klein-Gordon equation can be Fourier-decomposed into uncoupled normal modes with angular frequency $\omega_{k}$. This suggests an infinite collection of independent oscillators as a model of the classical field. The standard quantization of the simple harmonic oscillator deploys 'raising' and 'lowering' operators interdefined with $\hat{p}$ and $\hat{q}$ and facilitating a perspicuous treatment of system energetics. Exploiting the analogy with the simple harmonic oscillator, the textbook approach represents the CCRs quantizing Klein-Gordon theory by means of 'raising' and 'lowering' operators $\hat{a}_{k}^{\dagger}$ and $\hat{a}_{k}$ for positive frequency (with respect to inertial time $t$ ) field modes $\omega_{k}$ :

$$
\left[\hat{a}_{k}, \hat{a}_{k^{\prime}}\right]=0=\left[\hat{a}_{k}^{\dagger}, \hat{a}_{k^{\prime}}^{\dagger}\right], \quad\left[\hat{a}_{k}, \hat{a}_{k^{\prime}}^{\dagger}\right]=\delta_{k k^{\prime}} \hat{I}
$$

These operators (which are more commonly, albeit misleadingly (see Teller 1995), known as 'creation and 'annihilation' operators) act on a kind of Hilbert space known as a symmetric Fock space (see Wald 1994, A.2 for a definition).

The action of the raising and lowering operators facilitates the interpretation of quantized Klein-Gordon theory as particle physics. The Fock space features a state $|0\rangle$ such 
that $\hat{a}_{k}|0\rangle=0$ for all $k .|0\rangle$, called the vacuum state, is the lowest energy eigenstate of the quantum Hamiltonian for the free boson field (also handled by analogy with the simple harmonic oscillator). The state $\hat{a}_{k}^{\dagger}|0\rangle$ is an eigenstate of the Hamiltonian with the same total energy a particle with frequency $\omega_{k}$ would have - provided the momentum and rest masses of this particles are given by standard relativistic expressions. The pattern reiterates. The state $\left|n_{k}\right\rangle$, obtained by acting on the vacuum $n$ times with $\hat{a}_{k}^{\dagger}$ and normalizing, has the energy appropriate to $n$ particles of frequency $\omega_{k}$, and so on. Acting on $\left|n_{k}\right\rangle$ with $\hat{a}_{k}$ and normalizing yields a state with the energy appropriate to $n-1$ particles of frequency $\omega_{k}$, and so on. (It is important to emphasize that these are truths about the relations between Fock space vectors and raising and lowering operators. While the truths tempt an understanding of the action of the raising operator as an event of physical particle creation, that is an optional interpretive overlay to the mathematical facts.) The operator $\hat{N}_{k}=\hat{a}_{k}^{\dagger} \hat{a}_{k}$ is known as the number operator for particles of variety $k$. Its eigenvalues are natural numbers; the state $\left|n_{k}\right\rangle$ is an $\hat{N}_{k}$ eigenstate with eigenvalue $n$. Thus $\hat{N}_{k}$ is understood to count particles of variety $k$ : its expectation value in a state gives the mean number of particles of variety $k$ one would find in that state. Hence, $\hat{N}=\sum_{j} \hat{a}_{j}^{\dagger} \hat{a}_{j}$ is the total number operator. That for all $k,\left\langle 0\left|\hat{N}_{k}\right| 0\right\rangle=\langle 0|\hat{N}| 0\rangle=0$ reinforces $|0\rangle$ 's claim to be the vacuum or no-particle state.

States that admit analysis in terms of their particle contents are not isolated. Every state in the Fock space can be approximated to arbitrary precision by acting on $|0\rangle$ with polynomial combinations of raising and lowering operators.

Still, it must be noted just how radically a particle concept framed by QFT departs from our ingrained particulate expectations. Particles, as we think of them, are confined to finite, bounded regions of spacetime. A number of results (Malament 1996; Halvorson and Clifton 2002) suggest that such confinement cannot be had in QFT. Particles, as we think of them, possess what the scholastics called 'haecceity', Teller $(1995,17)$ 'primitive thisness,' and Redhead (1983, 59) 'transcendental identity.' An extensive literature (see Castellani (1998) for a sample) questions whether bosons and fermions can exhibit this and yet abide by the non-Maxwell-Boltzmann statistics characteristic of their aggregates. Particles, as we think of them, are persistent in their presence and inert in their absence. Excitations of the quantum field are subject to creation and annihilation; even the vacuum fluctuates (Redhead, op. cit.). Particles, as we think of them, come in sets with determinate cardinality. States of the quantum field need not be eigenstates of the total particle number operator, and thus (on the usual way of thinking about quantum systems) need not be states describing a determinate number of particles (Teller 1995, Ch. 2). These are all grounds for doubting that QFT is a theory of particles as we think of them, particles as well-localized, enduring, individuable gobbets of mass-energy. But they leave intact the possibility of a particle concept fundamental to QFT in something like the way position and momentum are fundamental to classical mechanics: just as a classical state can be completely characterized and distinguished from other classical states by specifying its position and momentum, so too a state of the quantum field can be completely characterized in terms of its particle contents.

Because the phase space of Klein-Gordon theory is not finite, the Stone-von Neumann theorem cannot vouch that this representation is unique up to unitary equivalence. Indeed, the CCRs for the Klein-Gordon field admit continuously many inequivalent representations. (See Garding and Wightman (1954) or Arageorgis (1995, Appendix C), for a construction of unitarily inequivalent Fock space representations that deploys a move similar to that used in Section 5.1 of Aspects of QFT, Part I to exhibit unitarily inequivalent models of the infinite spin chain.) One symptom of this unitary inequivalence is that the vacuum state of one of a 
pair of unitarily inequivalent representations can't be realized as a density operator state on the other representation.

There is a consortium of tacit assumptions that tends to frame foundational engagement with physics. I'll call this consortium 'extremism'. Extremism supposes that there exist fundamental, non-approximate laws that determine which circumstances are physically possible and which aren't. It supposes further that it is the task of physics to identify these laws, and identify them with precision: physics aims at truth outright, and nothing so hazy as approximate truth. Extremism accordingly supposes that to interpret a physical theory is to take its laws as these fundamental ones, and so to seek to characterize the unique and determinate set of physical possibilities those laws allow. What falls outside this set simply isn't physically possible, according to the theory so interpreted; what falls within is simply physically possible; because the laws are fundamental and exact, there is nothing in between. Here I will try to suggest that part of what makes $\mathrm{QM}_{\infty}$ interesting is the pressure it puts on these extremist assumptions about the character of physics and the task of interpretation. I will develop my suggestion by, until further notice, operating within the scope of these (now not so tacit) extremist assumptions, and identifying the limitations of interpretations of the sort considered.

So assume that theorists wielding inequivalent representations are extremists. Each assumes that the set of states countenanced by his or her representation coincides with the unique set of physically possible states. Then the theorists don't simply disagree about which state is the ground state of the free boson field. Each denies that the other's ground state is a state at all. That is, they're not disagreeing about how to describe worlds they both agree are possible. A translation manual of the sort set up by a unitary map between their theories could resolve (or at least drastically temper) that disagreement. They're disagreeing about which worlds are possible to begin with. Because the sets of worlds each deems possible are (in a sense that can be made precise) disjoint, theorists wielding unitarily inequivalent representations can't both be even partially right. Regarded as theories of ordinary QM, unitarily inequivalent representations of the CCRs quantizing classical Klein-Gordon theory are out-and-out rivals. This leaves us with continuum-many starkly distinct theories vying for the title of free boson field - a veritable embarrassment of riches for particle physics.

\section{Haag's Theorem: Particle Ontology}

So far, the QFTs under discussion have been free field theories. Real particle physics treats interactions, often through the so-called 'interaction picture'. To first approximation, the interaction picture works as follows. What we want is a scattering amplitude between incoming states $\left|\psi_{\text {in }}\right\rangle$ (e.g., the state of an electron-postiron pair) and outgoing states $\left|\phi_{\text {out }}\right\rangle$ (e.g., the state of a pair of muons). The gadget that predicts probabilities for this pair-production process is the S-matrix $S$, in the sense that

$$
\left\langle\psi_{\text {in }}|S| \phi_{\text {out }}\right\rangle
$$

gives us the quantum mechanical amplitude that a system enters the detector in the state $\left|\psi_{\text {in }}\right\rangle$ and leaves it in the state $\left|\phi_{\text {out }}\right\rangle$. Insofar as both $\left|\psi_{\text {in }}\right\rangle$ and $\left|\phi_{\text {out }}\right\rangle$ admit an interpretation in terms of particle contents, the event for which (3) gives the amplitude can be understood as a particle scattering event.

Assume that at early and late times - that is, $t= \pm \infty$ - the system is governed by a free Hamiltonian $H$. What generates interesting scattering amplitudes is the imposition of a non-zero interaction Hamiltonian $H_{\text {int }}$ at intermediate times. The interaction picture provides a recipe for expressing S-matrix elements such as $\left\langle\psi_{\text {in }}|S| \phi_{\text {out }}\right\rangle$ in terms of the free 
and interacting Hamiltonians (see Peskin and Schroeder 1995). Extending a particle interpretation of incoming and outgoing particles to particles at intermediate times $-\infty<t<\infty$, we assume that a single Fock space representation embraces them all.

Haag's theorem reveals this assumption to be false, at least if $H_{\text {int }}$ is different from 0 . To simplify somewhat (see Earman and Fraser 2006 for further discussion), Haag's theorem shows that if certain prima facie reasonable assumptions about the interacting theory are satisfied, then that theory is not really an interacting theory at all: it's a theory in which $H_{\text {int }}=0$ ! As a consequence, any non-trivial (that is, $H_{i n t} \neq 0$ ) interacting theory satisfying the assumptions is unitarily inequivalent to the free (that is, $H_{\text {int }}=0$ ) theory.

The importance of Haag's theorem is multifold. For one thing, it makes a physically compelling case for the relevance of unitarily inequivalent representations to the interpretation of QFT. For it suggests that if we want to contend with interactions, we must come to terms with unitarily inequivalent representations. For another thing, it represents a challenge to the idea that a single particle notion could be fundamental. Supposing that some particle notion is appropriate to the interacting theory (a supposition Fraser (2008) challenges), Haag's theorem alerts us that if the interaction is non-trivial, that particle notion is incommensurable with the particle notion appropriate to the free theory. The apparent upshot is that there isn't a single particle notion that embraces the entire history of a scattering experiment. Perhaps all is not so bleak for particles: Bain (2000) has explored options for making sense of particles in the context of interacting quantum field theories.

\section{Abstraction: Algebraic Approaches}

Investigations of the formal structure of standard realizations of quantum field theoretic CCRs conducted in the $50 \mathrm{~s}$ and $60 \mathrm{~s}$ made possible an intriguing commentary on their embarrassing abundance. Each concrete Hilbert space representation of the CCRs gives rise to an algebra, called the Weyl algebra, which is representation-independent. The Weyl algebra for a system is constructed by taking linear combinations of operators satisfying the CCRs for that system, then closing in the norm topology, whose criterion of convergence is stricter than that of the weak topology we use to build $\mathfrak{B}(\mathscr{H})$ from a representation of the CCRs. This Weyl algebra is unique in the sense that no matter what Hilbert space realization of the CCRs for the system one starts with, one obtains the same Weyl algebra. Thus independent of its Hilbert space antecedents, the Weyl algebra for a system can be regarded as an abstract algebra, the algebraic structure shared by all its Hilbert space representations. For a system whose canonical observables obey CARs, one can likewise abstract from those CARs a single algebraic structure common to every Hilbert space representation of those CARs. Technically, these algebras are $\mathrm{C}^{*}$ algebras; see Kadison and Ringrose (1983, Ch. 4) for a more formal introduction.

Although this algebraic structure can be considered in abstraction from any concrete Hilbert space, an abstract algebra $\mathfrak{U}$ will always admit a concrete Hilbert space representation, an algebraic-structure-preserving map $\pi$ from $\mathfrak{U}$ into $\mathfrak{B}(\mathscr{H})$. In particular, all Hilbert spaces carrying a representation of the Weyl relations are also representations of the abstract Weyl algebra. Two representations $(\pi, \mathscr{H})$ and $\left(\pi^{\prime}, \mathscr{H}^{\prime}\right)$ of the same algebra $\mathfrak{U}$ are unitarily equivalent just in case there exists a unitary map $U: \mathscr{H} \rightarrow \mathscr{H}^{\prime}$ such that for each $A \in \mathfrak{U}, U \pi(A) U^{-1}=\pi^{\prime}(A)$. A QFT operates outside the scope of the Stone-von Neumann theorem, and admits representations that fail to be unitarily equivalent.

The algebraic approach to quantum theories associates observables pertaining to a system with the self-adjoint elements of a $C^{*}$ algebra $\mathfrak{A}$ appropriate to the system. The 
algebraic approach accommodates ordinary QM's picture of observables as a special case: $\mathfrak{U}$ is set equal to $\mathfrak{B}(\mathscr{H})$ for some Hilbert space $\mathscr{H}$. In ordinary QM, states on $\mathfrak{B}(\mathscr{H})$ are normed, positive, countably additive linear functionals over that algebra. If $\operatorname{dim}(\mathscr{H})>2$, Gleason's theorem places states, so conceived, in one-to-one correspondence with density operators on $\mathscr{H}$. By contrast, the algebraic approach to QM defines states directly in terms of the observable algebra $\mathfrak{A}$, not assumed to coincide with some $\mathfrak{B}(\mathscr{H})$. An algebraic state $\omega$ on $\mathfrak{U}$ is a linear functional $\omega: \mathfrak{U} \rightarrow \mathbb{C}$ that is normed $(\omega(I)=1))$ and positive $\left(\omega\left(A^{*} A\right) \geq 0\right.$ for all $\left.A \in \mathfrak{U}\right)$. $\omega(A)$ may be understood as the expectation value of (self-adjoint) $A \in \mathfrak{U}$. Like the states of ordinary QM, the set of states on a $C^{*}$ algebra $\mathfrak{U}$ is convex. Its extremal elements - that is, states $\omega$ which cannot be expressed as non-trivial convex combinations of other states - are pure states; all other states are mixed.

Connections both straightforward and surprising can be established between the ordinary QM and the algebraic notions of state. Straightforwardly, an ordinary QM state $\hat{W}$ acting on a Hilbert space $\mathscr{H}$ carrying a representation $\pi: \mathfrak{U} \rightarrow \mathfrak{B}(\mathscr{H})$ of an algebra $\mathfrak{A}$ defines an algebraic state $\omega$ on $\mathfrak{A}$. Simply set $\omega(A)=\operatorname{Tr}(\hat{W} \pi(A))$ for all $A \in \mathfrak{A}$. Surprisingly, we can travel in the other direction, from an abstract algebraic state to its realization on a concrete Hilbert space. Let $\omega$ be a state on a $C^{*}$ algebra $\mathfrak{A}$. Then there exists a Hilbert space $\mathscr{H}_{\omega}$, a representation $\pi_{\omega}: \mathfrak{U} . \rightarrow \mathfrak{B}\left(\mathscr{H}_{\omega}\right)$ of the algebra, and a cyclic ${ }^{1}$ vector $\left|\Psi_{\omega}\right\rangle \in \mathscr{H}_{\omega}$ such that, for all $A \in \mathfrak{U}$, the expectation value the algebraic state $\omega$ assigns $A$ is duplicated by the expectation value the Hilbert space state vector $\left|\Psi_{\omega}\right\rangle$ assigns the Hilbert space observable $\pi(A)$. The triple $\left(\mathscr{H}_{\omega}, \pi_{\omega},\left|\Psi_{\omega}\right\rangle\right)$ is unique up to unitary equivalence, and known as the state's GNS (named for Gel'fand, Naimark, and Segal, who devised it) representation.

Clearly, a state $\omega$ on a algebra $\mathfrak{A}$ can, by the agency of the cyclic vector $\left|\Psi_{\omega}\right\rangle$, be expressed in terms of $\omega$ 's GNS representation. But $\omega$ is not the only algebraic state that has a natural home in that GNS representation. In particular, some algebraic states $\phi$ on $\mathfrak{U}$ can be implemented as density operator states on $\omega$ 's GNS representation, in the sense that there exists a density operator $\hat{W}$ acting on $\mathscr{H}_{\omega}$ such that $\phi(A)=\operatorname{Tr}\left(\hat{W} \pi_{\omega}(A)\right)$ for all $A \in \mathfrak{U}$. The set of algebraic states expressible as density matrices on $\omega$ 's GNS representation comprise what is known as $\omega$ 's folium. When the GNS representations $\pi_{\omega}$ and $\pi_{\phi}$ of two algebraic states are unitarily equivalent, the folia of those algebraic states coincide. If two pure algebraic states $\phi$ and $\omega$ have unitarily inequivalent GNS representations, their folia are disjoint: no algebraic state expressible as density matrix on $\phi$ 's GNS representation is so-expressible on $\omega$ 's and vice-versa. (With mixed algebraic states, the situation is more delicate. See Kadison and Ringrose (1983, Ch. 4) for details.) Disjoint states are states whose folia are disjoint.

The algebraic notions just sketched help to articulate, and to evaluate, strategies for interpreting $\mathrm{QM}_{\infty}$. To that task let us turn.

\section{Interpretation}

An extremist interpretation of a physical theory seeks to carve out the space of physical possibilities determined by the theory in one fell swoop, and by appeal only to the laws of the theory interpreted and other considerations (e.g., metaphysical, mathematical, maybe even methodological) similarly fundamental. Thus the output of an extremist interpretation of a theory $T$ is the unique and unambiguous set of circumstances simply physically possible according to $T$. This catalog of interpretive options begins with two extremisms that are liable to have already occurred to the reader. 


\section{I. HILBERT SPACE CONSERVATISM}

Following the precedent of ordinary QM, the interpreter I'll label the Hilbert space conservative identifies the kinematic content of a theory of $\mathrm{QM}_{\infty}$ the same way it's standardly identified on behalf of a theory of ordinary QM. The theory's observables form the selfadjoint part of $\mathfrak{B}(\mathscr{H})$ for some concrete separable Hilbert space $\mathscr{H}$; its states are density operators on that Hilbert space. The conservative thereby inherits a criterion of physical equivalence for theories of ordinary QM so understood: they're physically equivalent just in case they're unitarily equivalent.

The conservative's work hardly ceases here. In ordinary QM, the uniqueness theorems assure us, once the CCRs/CARs individuating a theory are fixed, all their representations are unitarily equivalent. This is not so in $\mathrm{QM}_{\infty}$. There the CCRs or CARs individuating a theory admit continuously many unitarily inequivalent Hilbert space representations. Committed to unitary equivalence as a criterion of physical equivalence, the Hilbert space conservative must regard each of these as rival quantum theories. At most one of them can be the theory she's set out to interpret. Thus the burden of the Hilbert space conservative is to articulate and motivate a principle of privilege that enables her to identify the single unitary equivalence class of physical representations, and reject the rest as without physical content.

Promising principles of privilege apply to quantum field theories, and appeal to the symmetries of the spacetimes in which they're set. In a spacetime equipped with global time symmetries, a unitary equivalence class of representations of CCRs for the KleinGordon field sustains an energy observable respecting those isometries. The respect takes roughly the following form: a privileged Hamiltonian operator can be identified as the infinitesmal generator of time translations that leave the metric unchanged. A respectful representation has a vacuum state which is not only itself invariant under these time symmetries, but also the lowest energy eigenstate of the privileged Hamiltonian. What's more, each $n$-particle state of a respectful representation is an eigenstate of the privileged Hamiltonian, with an eigenvalue matching the energy of a corresponding classical state. Kay and Wald (1991) show that in suitably symmetric spacetimes, respectful representations of the Klein-Gordon CCRs are unique up to unitary equivalence.

If it's reasonable to demand such respect, it's reasonable to distinguish invidiously between respectful and disrespectful representations. The invidious distinction makes available univocal and powerful particle talk: the distinguished Fock space representation circumscribes a (similarly distinguished) particle notion. Particle notions incommensurable with, because circumscribed by representations unitarily inequivalent to, that of the respectful representation can be dismissed as unphysical. Each state in the privileged folium can be characterized in terms of its particle content; different states can be distinguished in terms of their particle content. If an adequate interpretation is obliged to understand QFT as particle physics - and that is how many of its practitioners do understand it - then the pursuit of Hilbert space conservatism in spacetime settings with enough symmetry to pick out a particle-friendly unitary equivalence class of representations is one route to adequacy.

But QFT is also pursued in the unsymmetric curved spacetimes of general relativity, and these spacetimes block the route. Notoriously, the route is complicated even in a subset of Minkowski spacetime. Positive energy states correspond to solutions to the Klein-Gordon equation that oscillate with purely positive frequency. States in the standard Minkowski representation are positive frequency with respect to time as measured by families of inertial observers. But restricting our attention to the right Rindler wedge of two-dimensional 
Minkowski spacetime - using inertial coordinates and setting $c=1$, this is the wedgeshaped region to the right of the origin where $x$ is positive and less than $t-$ we can quantize the Klein-Gordon field by admitting solutions that oscillate with positive frequency with respect to time as measured by observers whose accelerations are constant. The Rindler representation we thereby obtain has a natural particle interpretation - but the Rindler representation is unitarily inequivalent to the Minkowski representation! This is sometimes, and loosely, expressed as the Unruh effect: observers accelerating through the Minkowski vacuum 'see' a thermal flux of particles (see Wald 1994, ch. 5). Some have regarded the availability, even within Minkowski spacetime, of incommensurable particle notions as grounds for demoting the particle notion from fundamental status (see Arageorgis et al. (2003) for a critical review). Clifton and Halvorson (2001) object that Minkowski and Rindler particle notions are better understood as 'complementary' than incommensurable. Picking up another thread of Copenhagen orthodoxy, Davies (1984) urges that the lesson of the Unruh effect is that the particle notion must be operationalized.

Quantizing in Rindler spacetime suggests that respect for symmetries may not be sufficient to privilege a unitary equivalence class of representations for a QFT. Baker (2008) has observed that respect for symmetries may not even always be desirable. So-called coherent states of the quantum field enjoy wide application in optics. But certain coherent states correspond to representations violating Poincaré symmetries! Insisting that physically reasonable representations exhibit symmetries removes these coherent states from the physicist's tool box.

When it comes to the thermodynamic limit of quantum statistical mechanics (QSM), Hilbert space conservatism threatens an even more drastic impoverishment of the theory's explanatory resources. Short of the thermodynamic limit, and in the setting of concrete Hilbert spaces, the Gibbs state equips QSM with a notion of equilibrium. For realistic, finite quantum systems this Gibbs state is well-defined and unique. For infinite quantum systems this needn't be so.

Suppose that we aspire to construct a quantum statistical account of phase structure. The apparent macroscopic explanandum is the existence, at certain temperatures, of multiple thermodynamic phases: reverting to the toy model of the infinite spin chain, it can enjoy a paramagnetic phase, heuristically modeled as a condition wherein its constituent spins are so disordered that they generate no net magnetic field, as well as ferromagnetic phases, heuristically modeled as conditions wherein its constituent spins are sufficiently aligned that a net magnetic field ensues. If an account of phase structure requires the existence, at these temperatures, of multiple distinct equilibrium states, answering to different thermodynamic phases, then, as long as we're dealing with finite systems, our explanatory hopes are dashed. At any given temperature, the Gibbs equilibrium state is unique, and so there is no temperature at which there are multiple distinct Gibbs states that might answer to different phases.

Explanatory hope is restored in the thermodynamic limit by using the KMS (named for Kubo, Marin, and Schwinger, who first remarked its connection to equilibrium) condition to explicate a notion of equilibrium more general than that afforded by the Gibbs state (see Ruetsche (2003) for elaboration and references). The set of KMS equilibrium states with respect to a given dynamics has several striking features. Consider a system whose observables belong to a $\mathrm{C}^{*}$ algebra $\mathfrak{A}$. Its equilibrium state at a temperature $T$ is disjoint from its equilibrium state at a different temperature. Provided neither temperature is 0 , each equilibrium state is moreover - and mind-bogglingly - disjoint from every pure state on $\mathfrak{U}$. And at critical temperatures where $\mathfrak{U}$ admits multiple KMS states, the extremal 
elements of that set - which correspond to the pure phases whose co-presence constitutes the phase structure we aspire to explain - are either equivalent or disjoint.

Hilbert space conservatism insists that at most one of a pair of disjoint representations of $\mathfrak{U}$ is the 'physical' representation coding genuine possibilities. By its lights, the rest are mathematical artifacts. But this implies that at most one equilibrium state at a non-zero temperature is possible. The implication hamstrings the attempt to explain phase structure in terms of the availability, at the critical temperatures at which phase transitions occur, of multiple distinct (and therefore disjoint) equilibrium states.

This challenge to Hilbert space conservatism take the form of charging that it recognizes too few states for physics to discharge its explanatory tasks. Next we consider an extreme reaction to this charge: to recognize as legitimate every algebraic state.

\subsection{ALGEBRAIC IMPERIALISM}

Inspired by the representation-independence of the Weyl algebra, an interpretive strategy Arageorgis (1995) has dubbed 'algebraic imperialism' construes a theory of $\mathrm{QM}_{\infty}$ not in terms of the Hilbert space setting of a particular concrete representation of the CARs/ CCRs for that theory, but in terms of the abstract algebraic structure every such representation shares. Physical magnitudes pertaining to a system are given by the self-adjoint part of the abstract algebra $\mathfrak{A}$; possible states are states in the algebraic sense on $\mathfrak{U}$. We are understanding this as a form of extremism: the algebraic imperialist declares once and for all, without qualification, what possibilities the theory allows; this declaration endows the theory with content.

Algebraic imperialism and Hilbert space conservatism are rival accounts of the content of $\mathrm{QM}_{\infty}$. For there can arise algebraic states $\omega$ and $\omega^{\prime}$ on an observable algebra $\mathfrak{U}$ of $\mathrm{QM}_{\infty}$ which are disjoint. Both are, according to the algebraic imperialist, possible states of the system associated with $\mathfrak{A}$. But there is no $\mathfrak{B}(\mathscr{H})$ generated by a concrete Hilbert space representation of $\mathfrak{A}$ on which both states can be implemented by density matrices. So there's no Hilbert space conservative account of the content of the theory according to which both states are possible. If unitary equivalence is criterial for physical equivalence, any theory admitting one state as possible will be physically inequivalent to a theory admitting the other as possible.

This makes it clear that unitary equivalence is a criterion of physical equivalence illadapted to algebraic imperialism. Recognizing a mismatch, early advocates of the algebraic approach offered instead of unitary equivalence weak equivalence as a criterion of physical equivalence for Hilbert space representations. Weak equivalence is a relation that holds (or not) between Hilbert space representations of a $\mathrm{C}^{*}$ algebra $\mathfrak{A}$. Two representations are weakly equivalent exactly when no finite set of expectation values of observables in $\mathfrak{U}$, specified to finite accuracy can locate a state in one, rather than the other, representation. The imperialist 'define[s] two representations to be physically equivalent if and only if they are weakly equivalent' (Robinson 1966: 488). A mathematical result known as Fell's theorem consolidates this definition. Fell's theorem implies that all faithful ${ }^{2}$ representations of a $C^{*}$ algebra are weakly equivalent (see Wald 1994, Ch. 4.5). Defining physical equivalence as weak equivalence, the imperialist can conclude that, 'All faithful representations of [the Weyl algebra] are physically equivalent' (Robinson 1966: 488).

Notice that the observables with respect to which weakly equivalent representations are practically indistinguishable are elements of the abstract algebra $\mathfrak{A}$. Consider, for some concrete representation $\left(\pi_{1}, \mathscr{H}_{1}\right)$ of $\mathfrak{U}$, an observable in $\mathfrak{B}\left(\mathscr{H}_{1}\right)$ but without correlate in $\mathfrak{H}$. I will call such an observable parochial to the representation $\pi_{1}$. Examples of parochial 
observables include the total number operators encountered in suitable representations of the Weyl algebra for the free Klein-Gordon field. The vacuum state of the $\pi_{1}$ representation assigns the total number operator expectation value 0 . But there is no density operator state in any representation $\pi_{2}$ unitarily inequivalent to $\pi_{1}$ that assigns this total number operator an expectation value within any finite $\epsilon$ of 0 (Clifton and Halvorson 2001, Prop. 11, 450). Every faithful representation of the Weyl algebra is weakly equivalent to every other. So imperialists would hail $\pi_{1}$ and $\pi_{2}$ as physically equivalent even though all $\pi_{2}$ states assign a $\pi_{1}$ observable - its total number operator - "expectation values' arbitrarily far from that assigned by the $\pi_{1}$ vacuum state. Of course, this is a compelling criticism of weak equivalence as an explication of physical equivalence only for those inclined - as imperialists are not - to regard parochial observables as physical.

The justifications imperialists offer for weak equivalence as a criterion of physical equivalence for Hilbert space representations are, broadly speaking, operationalist. But they ultimately fail, even by operationalist lights. For, as Summers (2001) has observed, even operationalists ought to take states to be predictive instrumentalities. Operationalists should therefore expect the equivalence of states to extend to their predictions concerning future measurements. But Fell's theorem offers us no assurance that a $\pi_{1}$ state for all practical purposes indistinguishable from a $\pi_{2}$ state with respect to some set $\left\{A_{i}\right\}$ of algebraic observables will continue to mimic the first state's predictions with respect to an expanded or altered set of observables.

Of course, the imperialist needn't nail his banner to the mast of weak equivalence which is, after all, a criterion of equivalence for Hilbert space representations, which representations the imperialist denies to be physically significant! A more direct imperialism would define quantum theories to be physically equivalent only if their associated algebras are isomorphic. Once an abstract algebra is identified as the algebra appropriate to a theory of $\mathrm{QM}_{\infty}$, this criterion of physical equivalence follows straightforwardly. The challenge for the bold imperialist is to justify his choice of algebra. Segal (1959), which develops an argument for the operational significance of norm but not (where they differ) weak closure, represents one attempt to rise to the challenge.

The Hilbert space conservative was liable to recognize too few states to support $\mathrm{QM}_{\infty}$ 's explanatory agendas. The imperialist is prone to the complementary shortfall of recognizing two few observables. As the discussion of weak equivalence suggests, the abstract algebra $\mathfrak{U}$ of observables an imperialist attributes a quantum field theoretic system typically excludes observables constituting a particle notion. The abstract algebra $\mathfrak{U}$ for a system at the thermodynamic limit of QSM excludes such significant observables as global charge, temperature, and chemical potential (Kronz and Lupher 2005). Reverting to the toy example of the infinite spin chain, the global polarization observable - the observable whose value distinguishes between the paramagnetic phase of no net magnetization and the ferromagnetic phase of net magnetization - is an observable from which the imperialist withholds physical significance. Broken symmetry is yet another phenomenon the toy model illustrates. On the supposition that the dynamics of the spin chain is rotationally symmetric, both the $\mathscr{H}^{+}$and $\mathscr{H}^{-}$representations break that symmetry. (The rotational symmetry is broken in the esoteric sense, elaborated in Earman (2004), of constituting a transformation that can't be implemented by a untiary operator on the representations in question.) What Liu and Emch (2005) have aptly termed a witness to broken symmetry is an observable that takes different values in different states of broken symmetry. Once again, global polarization, a magnitude the imperialist foreswears, is an example. Insofar as robust laws of physics involve commodities such as phase observables and witnesses to broken symmetry, imperialism denatures those laws. 


\subsection{SELECTION CRITERIA}

Some would temper unbridled imperialism by restricting admissible algebraic states, but not (necessarily) confining them to the constricted single folium favored by the Hilbert space conservative. I close by sketching some tempering strategies, and suggesting that we might see in these sketches reasons to abandon extremist interpretive policies.

\subsubsection{Dynamics}

So far we have focussed on the kinematics of theories of $\mathrm{QM}_{\infty}$. It could be that considerations of dynamics usefully constrain the set of physically relevant instantaneous states. Two prima facie reasonable constraints are that the set of physically relevant instantaneous states be closed under dynamical evolution, and that each state in this set be one for which dynamics are well-defined. The first constraint precludes a physically possible state evolving into a physically impossible one; the second builds a capacity for dynamical development into the very notion of physical possibility.

In ordinary Hilbert space QM, dynamics are typically implemented by unitary Hilbert space operators. In algebraic QM, dynamics are implemented by means of automorphisms of the algebra $\mathfrak{U}$ of observables (that is, structure-preserving maps from $\mathfrak{U}$ to itself). A question of equipollence arises: is it the case that every dynamical evolution implementable by an automorphism on the an algebra $\mathfrak{A}$ is also implementable as a unitary evolution in a concrete Hilbert space representation of that algebra? In QFT, the answer is no: dynamical automorphisms corresponding (in an admittedly attenuated sense) to time evolution between time slices related by isometries can be implemented unitarily in standard vacuum representations, but more general dynamical automorphisms cannot be (see Arageorgis et al. (2002), for details). In other words, a system starting in algebraic state $\omega$ can evolve into a state disjoint from $\omega$. A Hilbert space conservative regarding $\omega$ 's folium as the folium of physical possibilities must reject the endpoint of evolution as physical. Such rejection is sufficiently awkward to suggest that in more general settings the algebraic formulation is better suited to expressing QFT's dynamics.

Unitarity breaks down even more dramatically in the exotic spacetime setting of an evaporating black hole. Hawking has argued that a pure to mixed state transition - the sort of transition von Neumann's collapse postulate asserts to happen upon measurement - occurs in the course of black hole evaporation. Not only unitarity but also symmetries of time and pre/retrodiction are lost if Hawking is right. Belot et al. (1999) review reactions to the Hawking Information Loss Paradox; not the least of the many questions the Hawking paradox raises is how to pursue QFT in non-globally hyperbolic spacetimes.

Aristotle would advocate restricting physically admissible states to those that sustain dynamics. The cases to which this principle applies most directly come from the thermodynamic limit of quantum statistical mechanics. There it can happen that the abstract algebra $\mathfrak{A}$ sustains no dynamics of its own, but dynamics are well-defined on Hilbert spaces carrying particular concrete representations $\pi$ of $\mathfrak{U}$ (see Sewell 2002, \$2.4.5). The idea behind this principle is that states whose GNS representations enable a well-defined dynamics are physical; states that do not admit of dynamical development are not.

\subsubsection{The Hadamard condition}

Semiclassical quantum gravity considers a quantum field on spacetime manifold subject to Einstein's field equations, with a quantum commodity (the expectation value $\left\langle T_{a b}\right\rangle$ of the stress energy tensor $T_{a b}$ ) substituted for a classical one (plain old $T_{a b}$ ). A restriction that looks down the road to quantum gravity is the Hadamard condition, which requires 
admissible state to be states for which a prescription assigning the stress-energy tensor an expectation value succeeds. (Provocatively, in closed spacetimes such states form a unitary equivalence class (see Wald 1994, S 4.6).) But this principle stands in surprising tension with another heralded signpost along the road to quantum gravity: Hawking radiation and black hole entropy. The Unruh vacuum, which is the state some contend gives the best model of what happens outside an evaporating black hole, violates the Hadamard condition (Candelas 1980).

\subsubsection{DHR selection criteria}

DHR (Doplicher, Haag, and Roberts) selection theory is an approach to superselection in algebraic QFT which identifies the physical representations of the field algebra $\mathfrak{U}$ with those representations whose observable differences from the representation $\pi_{0}$ of a fixed vacuum state are confined to local regions. Considering the set of DHR representations from the vantage point of category theory, one can then reconstruct the quantum fields and the gauge group. For more about this powerful and profound approach, see Halvorson and Müger (2007) or Baker and Halvorson (2010). Abstracting away from the details, observe that this principle of selection proceeds from a pre-identified vacuum state. It is fair to ask what justifies the selection of this vacuum state as the reference state. It may be fair to answer that the explanatory accomplishments of the ensuing theory do!

\section{Conclusion}

Attempts to temper the extremisms of Hilbert space conservatism and algebraic imperialism were prompted by the inadequacy of those stances to the demands of physics. Each extremism undermined some application of the theory it would interpret, some use of that theory to explain, predict, or engender other theories. As cursory as the foregoing account of possible tempering principles is, it may still suggest that there isn't a single universal corrective for this: that under different applications, a typical theory of $\mathrm{QM}_{\infty}$ might call for differently tempered interpretations, so that no single interpretation makes univocal sense of that theory's galaxy of empirical and theoretical successes. If this is so, the tacit suppositions constituting extremism are incorrect. The content of a physical theory isn't given by a unique and unequivocal space of possibilities determined by that theory's fundamental laws and good taste, but rather by various sets, variously configured, sometimes with the exigencies of applications in mind. To my mind, the point of trying to interpret theories of $\mathrm{QM}_{\infty}$ is their capacity to dramatize the predicament that no lonely extremist interpretation can do them justice, and to thereby invite reflection - reflection across the foundations of all physics, not just $\mathrm{QM}_{\infty}-$ about the predicament's extent and significance.

\section{Acknowledgement}

I am grateful to the editors for their patience and to Paul Teller and other readers at UC-Davis for terrific feedback on an earlier draft.

\section{Short Biography}

Laura Ruetsche has been a professor of philosophy at the University of Michigan since 2008. She has also held tenure track appointments at the University of Pittsburgh (where she got her $\mathrm{PhD}$ under the direction of John Earman in 1995) and Middlebury College, 
as well as visiting appointments at Rutgers and Cornell. Her book, Interpreting Quantum Theories, has just been published by Oxford University Press.

\section{Notes}

* Correspondence: Department of Philosophy, University of Michigan, 435 S. State Str, Ann Arbor, MI 48109, USA. Email: ruetsche@pitt.edu.

$1|\Psi\rangle$ is cyclic for $\pi_{\omega}(\mathfrak{N})$ means $\left\{\pi_{\omega}(\mathfrak{A})|\Psi\rangle\right\}$ is dense in $\mathscr{H}$.

${ }^{2} \pi$ is faithful iff $\pi(A)=0$ implies $A=0$ for all $A \in \mathfrak{A}$.

\section{Works Cited}

Arageorgis, Aristitidis. Fields, Particles, and Curvature: Foundations and Philosophical Aspects of Quantum Field Theory in Curved Spacetime, Ph.D. Diss.. Pittsburgh, PA: University of Pittsburgh, 1995.

— John Earman, and Laura Ruetsche. 'Fulling Non-uniqueness, Rindler Quanta, and the Unruh Effect: A Primer on Some Aspects of Quantum Field Theory.' Philosophy of Science 70 (2003): 164-202.

— $—$ and — . 'Weyling the Time Away: The Non-unitary Implementability of Quantum Field Dynamics on Curved Spacetime and the Algebraic Approach to QFT.' Studies in the History and Philosophy of Modern Physics 32 (2002): 151-84.

Bain, J. 'Against Particle/Field Duality: Asymptotic Particle States And Interpolating Fields In Interacting Qft (Or: Who's Afraid of Haag's Theorem?).' Erkenntnis 53 (2000): 375-406.

Baker, David. 'Against Field Interpretations of Quantum Field Theory.' British Journal for the Philosophy of Science 60 (2008): 508-609.

— and Hans Halvorson. 'Anti-matter.' British Journal for the Philosophy of Science 61 (2010): 93-121.

Belot, G., J. Earman, and L. Ruetsche. 'The Hawking Information Loss Paradox: Anatomy of a Controversy.' British Journal for the Philosophy of Science 50 (1999): 189-229.

Candelas, P. 'Vacuum Polarization in Schwarzschild Spacetime.' Physical Review D 21 (1980): 2185-202.

Castellani, E., ed. Interpreting Bodies: Classical and Quantum Objects in Modern Physics. Princeton: Princeton UP, 1998.

Clifton, Robert and Hans Halvorson. 'Are Rindler Quanta Real? Inequivalent Particle Concepts in Quantum Field Theory.' British Journal for the Philosophy of Science 52 (2001): 417-70.

Davies, P. C. W. 'Particles Do Not Exist.' Quantum Theory of Gravity. Ed. S. M. Christensen. Bristol: Adam Hilger, 1984. 66-77.

Earman, John. 'Laws, Symmetry and Symmetry Breaking: Invariance, Conservation Principles, and Objectivity.' Philsophy of Science 71 (2004): 1227-41.

— and Doreen Fraser. 'Haag's Theorem and its Implications for the Foundations of Quantum Field Theory.' Erkenntnis 64 (2006): 305-44.

Fraser, Doreen. 'The Fate of "Particles" in Quantum Field Theories with Interactions.' Studies in the History and Philosophy of Modern Physics 39 (2008): 841-59.

Garding, L. and A. Wightman. 'Representations of the Commutation Relations.' Proceedings of the national Academy of the Sciences USA 10 (1954): 622-6.

Halvorson, H. and R. Clifton. 'No Place for Particles in Relativistic Quantum Theories.' Philosophy of Science 69 (2002): 1-28.

Halvorson, Hans and Michael Müger. 'Algebraic Quantum Field Theory.' Philosophy of Physics, Part A. Eds. Jeremy Butterfield and John Earman. Amsterdam: Elsevier, 2007. 731-922.

Kadison, R. V. and J. R. Ringrose. Fundamentals of the Theory of Operator Algebras, 1 vol. New York: Academic Press, 1983.

Kaku, M. Quantum Field Theory: A Modern Introduction. Oxford: Oxford University Press. 1993.

Kay, B. S. and R. M. Wald. 'Theorems on the Uniqueness and Thermal Properties of Stationary, Nonsingular, Quasifree States on Spacetimes with a Bifurcate Killing Horizon.' Physics Reports 207 (1991): 49-136.

Kronz, F. and T. Lupher. 'Unitarily Inequivalent Representations in Algebraic Quantum Theory.' International Journal of Theoretical Physics 44 (2005): 1239-58.

Liu, Chiang and Gérard Emch. 'Explaining Quantum Spontaneous Symmetry Breaking.' Studies in the History and Philosophy of Modern Physics 36 (2005): 137-63.

Malament, D. 'In Defense of Dogma.' Perspectives on Quantum Reality: Non-Relativistic, Relativistic, and Field-Theoretic. Ed. R. Clifton. Dordrecht: Kluwer, 1996. 1-10.

Peskin, Michael and Daniel Schroeder. An Introduction to Quantum Field Theory. Reading, MA: Addision-Wesley, 1995. 
Redhead, M. 'Quantum Field Theory for Philosophers.' PSA 1982. 2 vols. Eds. P. Asquith and T. Nickes. East Lansing, MI: Philosophy of Science Association, 1983. 57-99.

Robinson, D. W. 'Algebraic Aspects of Relativistic Quantum Field Theory.' Axiomatic Field Theory. Eds. M. Chretien and S. Deser. New York: Gordon and Breach, 1966. 391-516.

Ruetsche, Laura. 'A Matter of Degree: Putting Unitary Inequivalence to Work.' Philosophy of Science [Proceedings] 70 (2003): 1329-42.

Segal, I. E. 'The Mathematical Meaning of Operationalism in Quantum Mechanics.' Studies in Logic and the Foundatios of Mathematics. Eds. L. Henkin, P. Suppes, and A. Tarski. Amsterdam: North-Holland, 1959. 341-52.

Sewell, Geoffrey. Quantum Mechanics and its Emergent Metaphysics. Princeton, NJ: Princeton University Press, 2002.

Summers, Stephen. 'On the Stone-von Neumann Uniqueness Theorem and its Ramifications.' John von Neumann and the Foundations of Quantum Physics (Budapest, 1999). Vienna Circle Institute Yearbook, 8. Eds. Miklós Rédei, Michael Stöltzner. Dordrecht: Kluwer, 2001. 135-52.

Teller, Paul. An Interpretive Introduction to Quantum Field Theory. Princeton, NJ: Princeton UP, 1995.

Wald, Robert M. General Relativity. Chicago: U of Chicago P. 1984.

—. Quantum Field Theory in Curved Spacetime and Black Hole Thermodynamics. Chicago: U of Chicago P, 1994. 